\title{
Novel dual-color immunohistochemical methods for detecting ERG-PTEN and ERG-SPINK1 status in prostate carcinoma
}

Ritu Bhalla ${ }^{1,2}$, Lakshmi P Kunju ${ }^{1,2,3}$, Scott A Tomlins ${ }^{1,2}$, Kelly Christopherson ${ }^{4}$, Connie Cortez ${ }^{4}$, Shannon Carskadon ${ }^{1,2}$, Javed Siddiqui ${ }^{1,2}$, Kyung Park ${ }^{5}$, Juan Miguel Mosquera ${ }^{5}$, Gary A Pestano ${ }^{4}$, Mark A Rubin ${ }^{5}$, Arul M Chinnaiyan ${ }^{1,2,3,6,7}$ and Nallasivam Palanisamy ${ }^{1,2,3}$

${ }^{1}$ Michigan Center for Translational Pathology, Ann Arbor, MI, USA; ${ }^{2}$ Department of Pathology, University of Michigan, Ann Arbor, MI, USA; ${ }^{3}$ Comprehensive Cancer Center, University of Michigan, Ann Arbor, MI, USA; ${ }^{4}$ Ventana Medical Systems, A Member of the Roche Group, Tucson, AZ, USA; ${ }^{5}$ Department of Pathology and Laboratory of Medicine, Weill Cornell Medical College, New York, NY, USA; ${ }^{6}$ Department of Urology, University of Michigan, Ann Arbor, MI, USA and ${ }^{7}$ Howard Hughes Medical Institute, Chevy Chase, MD, USA

Identification of new molecular markers has led to the molecular classification of prostate cancer based on driving genetic lesions. The translation of these discoveries for clinical use necessitates the development of simple, reliable and rapid detection systems to screen patients for specific molecular aberrations. We developed two dual-color immunohistochemistry-based assays for the simultaneous assessment of ERGPTEN and ERG-SPINK1 in prostate cancer. A total of 232 cases from 184 localized and 48 metastatic prostate cancers were evaluated for ERG-PTEN and 284 cases from 228 localized and 56 metastatic prostate cancers were evaluated for ERG-SPINK1. Of the 232 cases evaluated for ERG-PTEN, 81 (35\%) ERG-positive and 77 (33\%) PTEN-deleted cases were identified. Of the 81 ERG-positive cases, PTEN loss was confirmed in 35 (15\%) cases by fluorescence in situ hybridization (FISH). PTEN status was concordant in 203 cases (sensitivity $90 \%$ and specificity $87 \% ; P<0.0001$ ) by both immunohistochemisty and FISH; however, immunohistochemisty could not distinguish between heterozygous and homozygous deletion status of PTEN. Of the 284 cases evaluated for ERG-SPINK1, $111(39 \%)$ cases were positive for ERG. In the remaining 173 ERG-negative cases, SPINK1 was positive in $26(9 \%)$ cases. SPINK1 expression was found to be mutually exclusive with ERG expression; however, we identified two cases, of which one showed concomitant expression of ERG and SPINK1 in the same tumor foci, and in the second case ERG and SPINK1 were seen in two independent foci of the same tumor nodule. Unlike the homogenous ERG staining in cancer tissues, heterogeneous SPINK1 staining was observed in the majority of the cases. Further studies are required to understand the molecular heterogeneity of cases with concomitant ERG-SPINK1 expression. Automated dual ERG-PTEN and ERG-SPINK1 immunohistochemisty assays are simple, reliable and portable across study sites for the simultaneous assessment of these proteins in prostate cancer.

Modern Pathology (2013) 26, 835-848; doi:10.1038/modpathol.2012.234; published online 25 January 2013

Keywords: fluorescent in situ hybridization; immunohistochemistry; prostate cancer; tissue microarray

Prostate cancer poses a major public health challenge for men in the United States and the

Correspondence: Dr N Palanisamy, PhD, Michigan Center for Translational Pathology, Department of Pathology, Comprehensive Cancer Center, University of Michigan Medical School, 2900, Huron Parkway, Traverwood IV, Suite 100, Room 1219, Ann Arbor, MI 48105, USA.

E-mail: nallasiv@med.umich.edu

Received 27 August 2012; revised 28 November 2012; accepted 3

December 2012; published online 25 January 2013 identification of disease-specific biomarkers is critical for the clinical management of prostate cancer. Over the past decade, several studies have identified various molecular aberrations in prostate cancer. Recurrent gene fusions involving the E26 transformation-specific (ETS) family of transcription factors, $E R G, E T V 1, E T V 4$ and ETV5, fused to androgenregulated TMPRSS2 and other $5^{\prime}$ partner genes have been identified in the majority of prostate cancers. ${ }^{1-8}$ The discoveries of gene fusions as well as other molecular lesions have potential 
implications for diagnosis, prognosis and therapy. ${ }^{1,8,9}$ Overall, early- and mid-stage localized prostate cancers and hormone-refractory metastatic cancers harbor TMPRSS2-ERG rearrangements in $\geq 50 \%$ cases, whereas high-grade prostatic intraepithelial neoplasia has a lower frequency of gene fusions $(10-20 \%) .{ }^{10-15}$ Benign prostate epithelial glands, atrophy or stroma do not demonstrate any expression of the $E R G$ gene fusion product, as reported by Perner et al, ${ }^{14}$ as well as other studies that used fluorescence in situ hybridization (FISH) to determine ERG rearrangement status. ${ }^{8,16}$

Genetic aberrations in nearly $50 \%$ of the remaining ETS-fusion-negative prostate cancer cases are largely unknown. Our group reported the identification of SPINK1 (serine peptidase inhibitor, Kazal type 1) overexpression in a subset of prostate cancer $(\sim 10 \%)$ that is mutually exclusive from ETS gene fusion-positive prostate cancers. ${ }^{17}$ In a subsequent study, Ateeq et $a l^{18}$ reported that the oncogenic phenotype mediated by SPINK1 overexpression can be inhibited by anti-SPINK1 antibody but had no effect on ERG gene fusion-mediated cell growth and metastasis, suggesting a potential therapeutic avenue for a subset of prostate cancer with SPINK1 overexpression. Another study to identify driving genetic aberrations in ETS fusion-negative prostate cancer using next-generation sequencing techniques led to the discovery of recurrent RAF (BRAF and $R A F 1)$ gene rearrangements in $1-2 \%$ of prostate cancers and subsets of gastric cancer and melanoma. These cancers harboring $B R A F$ and $R A F 1$ gene fusions can be targeted with approved and investigational drugs, the latter in late-stage development, and hence screening patients for these fusions will help identify those who may benefit from RAF kinase inhibitors. ${ }^{19}$

PTEN (phosphatase and tensin homolog deleted on chromosome 10) is a key tumor-suppressor gene in prostate cancer ${ }^{20}$ that plays an important role in the modulation of the phospatidylinositol-3-kinase (PI3K) pathway and downstream protein kinase, $A K T$. This pathway regulates a number of target genes such as BAD, CASP3 and CASP9, MDM2, mTOR, the forkhead family of transcription factors $(F K H R)$ and p27 that are involved in apoptosis and cell cycle progression. ${ }^{21-28}$ PTEN loss and subsequent activation of the PI3K pathway are associated with tumor progression in prostate cancer, ${ }^{29,30}$ and several new therapies targeting the P13K/AKT including inhibitors of mTOR, P13K and MEK (mitogen-activated kinase) are available. PTEN loss represents another molecular subset of prostate cancer; therefore, an accurate assessment of PTEN status in patients is important for pursuing appropriate therapies.

Although androgen-induced ETS gene fusionpositive tumors are associated with aggressive prostate cancer, both positive and negative correlations have been reported for gene fusions in prostate cancer. ${ }^{13,31,32}$ Several studies, including a population-based study, ${ }^{31}$ have found associations on univariate or multivariate analysis between ETS fusions and features of aggressive prostate cancer including higher Gleason grade, increased stage or decreased prostate-specific antigen recurrencefree survival. ${ }^{13,32-36}$ Other studies have reported no association with aggressive features or recurrence-free survival,32,37-41 whereas others found association with lower Gleason grade $^{39,42}$ or increased recurrence-free survival. ${ }^{43}$ However, concurrent PTEN loss and ERG rearrangements are generally associated with an aggressive phenotype. ${ }^{44}$

On the basis of these discoveries, Rubin et $a l^{45}$ developed a molecular classification system for prostate cancer comprising three categories: (1) prostate cancer with fusions involving ETS gene family members (2) prostate cancer with RAF kinase family fusions and (3) SPINK1-positive prostate cancers. The translation of these discoveries for clinical use necessitates the development of simple, reliable and rapid detection systems to screen patients for specific molecular biomarkers that are potential 'druggable' target. Until recently, FISH and PCR were the predominant methods for detection of most of the markers in prostate cancer. However, the availability of high-specificity antibodies affords us the opportunity to develop new approaches that are more clinically feasible and cost effective.

ERG rearrangements and PTEN loss have traditionally been detected by FISH studies using dualcolor break-apart and locus-specific probes, respectively, which are expensive, time consuming and require independent assessment on two separate slides. Given the multifocal nature of prostate cancer, methods that can simultaneously assess ERG and PTEN status as well as ERG and SPINK1 status would be ideal. Studies have reported high concordance between ERG immunohistochemistry and FISH in detecting ERG rearrangements, ${ }^{46,47}$ and high sensitivity of PTEN immunohistochemisty in detecting PTEN genomic loss. ${ }^{48,49}$ Determination of ERG, PTEN and SPINK1 status in independent parallel assays are cumbersome, time consuming and sometimes limited because of availability of tumor specimens, particularly tissues obtained from needle biopsy. Therefore, to overcome the technical limitations associated with independent assessment of ERG, PTEN and SPINK1 status in prostate cancer, we developed novel automated dual-color immunohistochemisty assays for the simultaneous assessment of ERG-PTEN and ERG-SPINK1 in prostate cancer and identified a new rare molecular subtype of prostate cancer with concomitant expression of ERG and SPINK1 in either the same or in different foci of same tumor nodule of prostate cancer. This assay is robust, easily portable to other laboratories and can be incorporated at numerous clinical sites to accommodate screening of large patient cohorts. 


\section{Materials and methods}

\section{Tissue Selection}

Multiple tissue microarrays were used in this study including cases from prostate and distant metastases collected at the University of Michigan Health System (details of tissue microarrays are provided in Table 1). The metastatic prostate carcinoma samples were obtained from patients diagnosed with hormone-refractory prostate cancer who were part of our posthumous tissue donor program. To date, 60 such autopsies have been performed. Normal and malignant tissues from multiple sites including bone were collected and incorporated in tissue microarrays used in this study.

The localized prostate cancer samples included radical prostatectomy cases with outcome information, various Gleason scores (screening tissue microarray) as well as prostate cancer cases with low PSA, rare morphologic variants and salvage prostatectomy cases. Although these are not consecutive radical prostatectomy cases, we are comfortable that they include the spectrum of localized prostate cancer cases seen at a high-volume institution that are not preselected.

In addition, the percentage of $E R G$-positive cases in these tissue microarrays (35-39\%), which have also been evaluated and confirmed for TMRPSS2$E R G$ fusion status by FISH using break-apart ERG assays as previously described, ${ }^{33,48}$ are similar to published data from prostatectomy series from other similar institutions. We are confident that this cohort is a representative of PSA-screened prostate cancers at a large tertiary academic center.

For the ERG-PTEN dual immunohistochemistry screening, a total of 232 evaluable cases were used in the analysis. Similarly, a total of 283 evaluable cases were used for the ERG-SPINK1 dual immunohistochemistry analysis. One independent case from Ventana Medical Systems (a member of the Roche Group) tumor bank was also included in the analysis. Patients either underwent radical prostatectomy or surgical resections of their metastatic lesions were included. On an average, 3 cores $(0.6 \mathrm{~mm})$ were obtained from each sample.

\section{Immunohistochemistry}

ERG-PTEN dual immunohistochemisty was performed using anti-ERG (EPR3864) rabbit monoclonal

Table 1 Types of tissue microarrays and distribution of cases for ERG-PTEN study

\begin{tabular}{lc}
\hline Types of TMA & $\begin{array}{c}\text { Number } \\
\text { of patients }\end{array}$ \\
\hline Low PSA PCA & 25 \\
Localized PCA & 69 \\
Progression including metastatic PCA & 53 \\
Salvage prostatectomy including metastatic PCA & 51 \\
Metastatic PCA & 34 \\
\hline
\end{tabular}

primary antibody (1:100; Cat no. 790-4576, Ventana Medical Systems, Tucson, AZ, USA) and a rabbit monoclonal primary antibody against PTEN (1:25; 138G6- Cell Signaling Technology, Danvers, MA, USA). Dual immunohistochemistry was performed using an automated protocol developed for the DISCOVERY XT automated slide staining system (Ventana Medical Systems) using UltraMap antirabbit HRP (Cat no. 760-4315, Ventana Medical Systems) for ERG and UltraMap anti-rabbit AP (Cat no. 760-4314, Ventana Medical Systems) for PTEN as secondary antibodies and were detected using ChromoMap DAB (Cat no. 760-159, Ventana Medical Systems) and ChromoMap Blue (Cat no. 760-161, Ventana Medical Systems) for ERG and PTEN, respectively. Nuclear Fast Red counterstain (Cat no. 780-2186 Ventana Medical Systems) was used as the counterstain. ERG-PTEN immunohistochemistry staining was evaluated by pathologists $\mathrm{RB}$ and LPK.

ERG-SPINK1 dual immunohistochemistry was performed using anti-ERG (EPR3864) rabbit monoclonal primary antibody (1:100; Cat no. 790-4576, Ventana Medical Systems) and a mouse monoclonal primary antibody against SPINK1 (1:100; Cat no. Abnova 24-80, Taipei City, Taiwan). Dual immunohistochemistry was performed using an automated protocol developed for the DISCOVERY XT automated slide staining system (Ventana Medical Systems) using UltraMap anti-rabbit HRP (Cat no. 760-4315, Ventana Medical Systems) for ERG and UltraMap anti-mouse AP (Cat no. 760-4312, Ventana Medical Systems) antibodies for SPINK1 as secondary antibodies and were detected using ChromoMap DAB (Cat no. 760-159, Ventana-Roche, Tucson, AZ, USA) and ChromoMap Red kit (Cat no. 760-160, Ventana-Roche) for ERG and SPINK1, respectively. Hematoxylin II (Cat no. 790-2208 Ventana-Roche) was used as counterstain. ERG and SPINK1 immunohistochemistry staining was evaluated by pathologists RB and LPK.

\section{Immunohistochemistry Evaluation Criteria}

Staining of vessels with nuclear expression was used as a positive control. ERG staining in prostatic glands was either absent or diffusely strong $(2-3+)$, unless otherwise indicated, and was reported as present/absent. Cores not displaying staining of vessels were classified as the 'antibody did not work' group. In addition, we used known ERG rearrangement (confirmed by FISH)-positive prostate cancer samples as positive control. Highgrade prostate intraepithelial neoplasia and lymphocytes also stained positive with the ERG antibody (lymphocytes usually demonstrated weak to moderate ERG positivity).

Cytoplasmic PTEN staining was observed in all benign prostatic glandular tissue including the basal epithelium. The fibromuscular stroma was negative 
for PTEN expression. A binary scoring system was applied for PTEN staining. The staining of tumor was compared with the benign epithelium and was scored as positive (increased or equal staining as compared with adjacent benign acini) or negative (decreased or absent staining). We defined staining as positive for PTEN when majority of cells ( $>90 \%$ ) showed PTEN staining; staining was defined as negative when it was either absent or weak staining in $<10 \%$ of cells. PTEN immunohistochemistry results were further validated by simultaneous FISH studies on the tissue microarrays.

SPINK1 expression in prostate cancer samples has been shown to be heterogeneous in previous studies $^{17,50}$ and also in our experience (unpublished observations of stained 60 prostate needle biopsies immunostained with SPINK1 IHC; Supplementary Figure 1). In this study, while evaluating the tissue microarray cores, only cytoplasmic staining within the cancerous epithelial cells were considered positive. Cytoplasmic SPINK1 expression was estimated and assigned values of $0 \%, 5 \%$ or multiples of $10 \%$. Any score above $5 \%$ was considered positive for SPINK1 expression. The fibromuscular stroma was negative for SPINK1 expression.

\section{Fluorescence In Situ Hybridization: ERG-PTEN}

BAC clones were used to generate the dualcolor break-apart FISH probes for ERG (RP11476D17-3' probe; RP11-95I21-5' probe), PTEN locus-specific probe (RP11-165M8) and chromosome 10 control probe (RP11-351D16). All clones were tested on normal human metaphase chromosomes to validate map position and these clones have been used extensively in various studies from our laboratory and others. ${ }^{7,51} 5^{\prime} E R G$ and chromosome 10 control probes were detected with antidigoxigenin fluorescein $\mathrm{Fab}$ fragments to yield green color and $3^{\prime} E R G$ probe and PTEN locus probes were detected with Streptavidin Alexa fluor 594 to yield red color. Based on the study reported by Park et $a l,{ }^{46}$ where we have shown concordance between ERG immunohistochemistry and FISH, confirmatory FISH for ERG was not performed.

\section{BAC DNA Preparation}

For each BAC clone, $200 \mathrm{ml}$ overnight cultures were grown in LB medium containing $12.5 \mu \mathrm{g} / \mathrm{ml}$ of chloramphenicol at $37^{\circ} \mathrm{C}$ for $14-16 \mathrm{~h}$ with constant shaking. DNA was prepared using Qiagen-midiprep kit using Qiatip-100 according to the protocol provided by the manufacturer (Qiagen, Valencia, CA, USA).

\section{Probe Labeling}

All FISH probes were prepared by nick translation labeling using modified nucleotides conjugated with biotin or digoxigenin utilizing biotin nick translation mix (11745824910, Roche, Indianapolis, IN, USA) for $3^{\prime}$ ERG and PTEN locus probes; digoxigenin nick translation mix (11745816910, Roche) for $5^{\prime} E R G$ and chromosome 10 control probes. Probe DNA was precipitated and dissolved in hybridization mixture containing $50 \%$ formamide, $2 \times$ SSC, $10 \%$ dextran sulfate and $1 \%$ Denhardt's solution. Approximately $200 \mathrm{ng}$ of each labeled probe was used for hybridization. Fluorescent signals were detected with Streptavidin Alexa fluor 594 (S-32356, Invitrogen, Carlsbad, CA, USA) and anti-digoxigenin fluorescein $\mathrm{Fab}$ fragments (11207741910, Roche) for red and green colors, respectively.

\section{Image Capture and FISH Signal Analysis}

FISH scoring was performed by an experienced cytogeneticist (NP) and a pathologist (RB). ERG rearrangement by translocation and/or deletion was recorded when the corresponding abnormal signal pattern was observed in $>10-15 \%$ of cells. Heterozygous deletion for PTEN was recorded when the cells contained one signal for the locus probe and two or more signals for the control probe compared with normal cells with two green and two red signals. Homozygous deletions were recorded when the cells contained no signal for the PTEN locus probe but two or more signal for the control probe. Fluorescent images were captured using a highresolution CCD camera controlled by ISIS image processing software (Metasystems, Altlussheim, Germany).

FISH scoring for ERG and PTEN was performed manually under $100 \times$ oil immersion objective in non-overlapping and morphologically intact nuclei. A minimum of 50 cells were scored from the cancer tissue. Areas of cancer tissue with weak or no signals and benign adjacent areas were not included in the analysis. For $E R G$, the normal signal pattern was recorded by the presence of a pair of colocalizing green and red signals and $5^{\prime}$ deletions were recorded by the presence of one colocalizing green and red (yellow) and one individual red signal. ERG translocations were recorded by the presence of one colocalizing green and red signal (yellow) and one non-colocalizing individual green and red signal. Based on the evaluation of the probes on normal prostatectomy FFPE specimens, we established a cutoff of $\geq 15-20 \%$ cells with the expected signal pattern for deletion, and translocations were recorded as positive. For PTEN, normal signal pattern was recorded by the presence of separate two green and two red signals for chromosome 10 control and PTEN locus probes, respectively. Hemizygous deletions were recorded with $>50 \%$ of cells containing one signal for the locus probe and $\geq 2$ signals for the chromosome 10 control probe. Homozygous deletions were recorded by the loss of both copies of 
PTEN locus probe and the presence of $\geq 2$ signals for chromosome 10 control probe in $>30 \%$ of cells as cutoff. Considering the sectioning artifacts, we established the cutoff values based on the evaluation on normal and tumor samples.

\section{Results}

\section{ERG-PTEN Dual-Color Immunohistochemisty Assay}

We performed dual-color ERG-PTEN immunohistochemistry in a wide spectrum of prostate tumors from 232 patients represented in tissue microarrays (Table 1). Of the 232 cases evaluated (184 localized prostate cancer and 48 metastatic prostate cancer; Table 2), 77 (33\%; 53 localized prostate cancer and 24 metastatic prostate cancer) cases with PTEN deletion were identified by immunohistochemisty. A small fraction of the tumors showed intratumoral heterogeneity for PTEN expression, with some areas staining positive for PTEN expression, whereas other areas were negative.

PTEN status by immunohistochemisty was further validated by FISH on all 232 cases (184 localized and 48 metastatic prostate cancers). Overall, concordance between negative immunohistochemisty and FISH signal indicating PTEN loss and positive immunohistochemisty and FISH signal indicating intact PTEN were identified in 203 cases (88\%). The specificity and sensitivity were $90 \%$ and $87 \%$, respectively $(P<0.0001$; Table 3$)$. A total of 142 cases (61\%) with no PTEN deletion by FISH and positive immunohistochemisty (true negative; Figure 1a and b), 22 cases (9\%) with no PTEN deletion by FISH but negative immunohistochemisty (false positive), 7 cases (3\%) with PTEN deletion by FISH but positive immunohistochemisty staining (false negative) and 61 cases $(27 \%)$ with PTEN deletion by FISH and negative immunohistochemisty (true positive) were observed (Figure 1c, d, g and h; Table 3). In all, 68 (29\%) cases (40 localized and 28 metastatic prostate cancers) with confirmed PTEN deletion were identified by FISH. However, immunohistochemisty was not consistent in separating heterozygous (36 cases, 16\%; Figure 1i and j) from homozygous loss (32 cases, 13\%; Figure 1g and $h)$.

Table 2 ERG and PTEN status

\begin{tabular}{lccc}
\hline ERG and PTEN status & $\begin{array}{c}\text { Localized } \\
\text { cancer }\end{array}$ & $\begin{array}{c}\text { Metastatic } \\
\text { cancer }\end{array}$ & $\begin{array}{c}\text { Total no. of } \\
\text { cases }\end{array}$ \\
\hline $\begin{array}{l}\text { Total number of cases } \\
\begin{array}{l}\text { ERG positive } \\
\text { PTEN deletion }\end{array}\end{array}$ & 184 & 48 & 232 \\
Immunohistochemisty & $53(37 \%)$ & $13(27 \%)$ & $81(35 \%)$ \\
FISH & $40(22 \%)$ & $24(50 \%)$ & $77(33 \%)$ \\
Heterozygous & $23(13 \%)$ & $13(27 \%)$ & $36(16 \%)$ \\
$\begin{array}{l}\text { Homozygous } \\
\text { ERG positive and PTEN }\end{array}$ & $17(9 \%)$ & $15(31 \%)$ & $32(13 \%)$ \\
deletion (by FISH) & $23(13 \%)$ & $12(25 \%)$ & $35(15 \%)$ \\
\end{tabular}

A total of 81 ERG (35\%)-positive cases were identified by immunohistochemistry, including 68 (37\%) localized and $13(27 \%)$ metastatic prostate carcinomas. No ERG staining was observed in benign prostatic glands. Simultaneous ERG rearrangement and PTEN deletion (as confirmed by FISH; Figure 1c and d) were identified in 35 cases including 23 localized and 12 metastatic prostate carcinomas and normal PTEN copies by FISH and immunohistochemisty with ERG rearrangement (Figure 1e and f) were observed in the remaining cases. Three cases demonstrated heterogeneous ERG expression.

\section{ERG-SPINK1 Dual-Color Immunohistochemisty Assay}

A total of 227 localized and 56 metastatic prostate carcinomas represented in tissue microarrays and one independent localized prostate cancer were used in the ERG-SPINK1 dual immunohistochemical assay. The details of the tissue microarrays are presented in Table 4.

In all evaluable cores, vascular endothelial cells and/or lymphocytes stained positive for ERG protein. A total of 111 ERG-positive cases were identified including 95 (42\%) localized and 16 $(29 \%)$ metastatic prostate carcinomas (Table 5). Out of 111 ERG-positive patients, 3 demonstrated heterogeneous staining whereas the remaining 108 patients had uniform staining in cancer tissues (Figure 2a and b). In one patient, ERG heterogeneity was recorded between discrete tumor nodules within the prostate gland; in a second patient, ERG heterogeneity was observed between metastatic sites. A third patient demonstrated ERG staining at one metastatic nodule in the liver, whereas all the other metastatic sites, including the second liver metastases, were negative.

Cancer-specific heterogeneous/multifocal pattern of cytoplasmic expression of SPINK1 was observed in all positive samples (Figure 2c and d). Mutual exclusivity was observed between ERG and SPINK1 antibody expressions except for two cases, as

Table 3 Concordance of PTEN expression by immunohistochemisty and FISH

\begin{tabular}{lr}
\hline & $\begin{array}{c}\text { Total no. of } \\
\text { cases }\end{array}$ \\
\hline $\begin{array}{l}\text { Concordant cases including true positive and true } \\
\text { negatives (immunohistochemisty/FISH) }\end{array}$ & $203(88 \%)$ \\
$\begin{array}{l}\text { PTEN deletion by FISH, negative PTEN } \\
\text { immunohistochemisty (true positive) }\end{array}$ & $61(27 \%)$ \\
$\begin{array}{l}\text { PTEN deletion by FISH, positive PTEN } \\
\text { immunohistochemisty staining (false negative) }\end{array}$ & $7(3 \%)$ \\
$\begin{array}{l}\text { No PTEN deletion by FISH, positive } \\
\text { immunohistochemisty staining (true negative) }\end{array}$ & $142(61 \%)$ \\
$\begin{array}{l}\text { No PTEN deletion by FISH, negative } \\
\text { immunohistochemisty staining (false positive) }\end{array}$ & $22(9 \%)$ \\
\hline
\end{tabular}

${ }^{\mathrm{a}}$ Sensitivity was $90 \%$ and specificity was $87 \%(P<0.0001)$. 

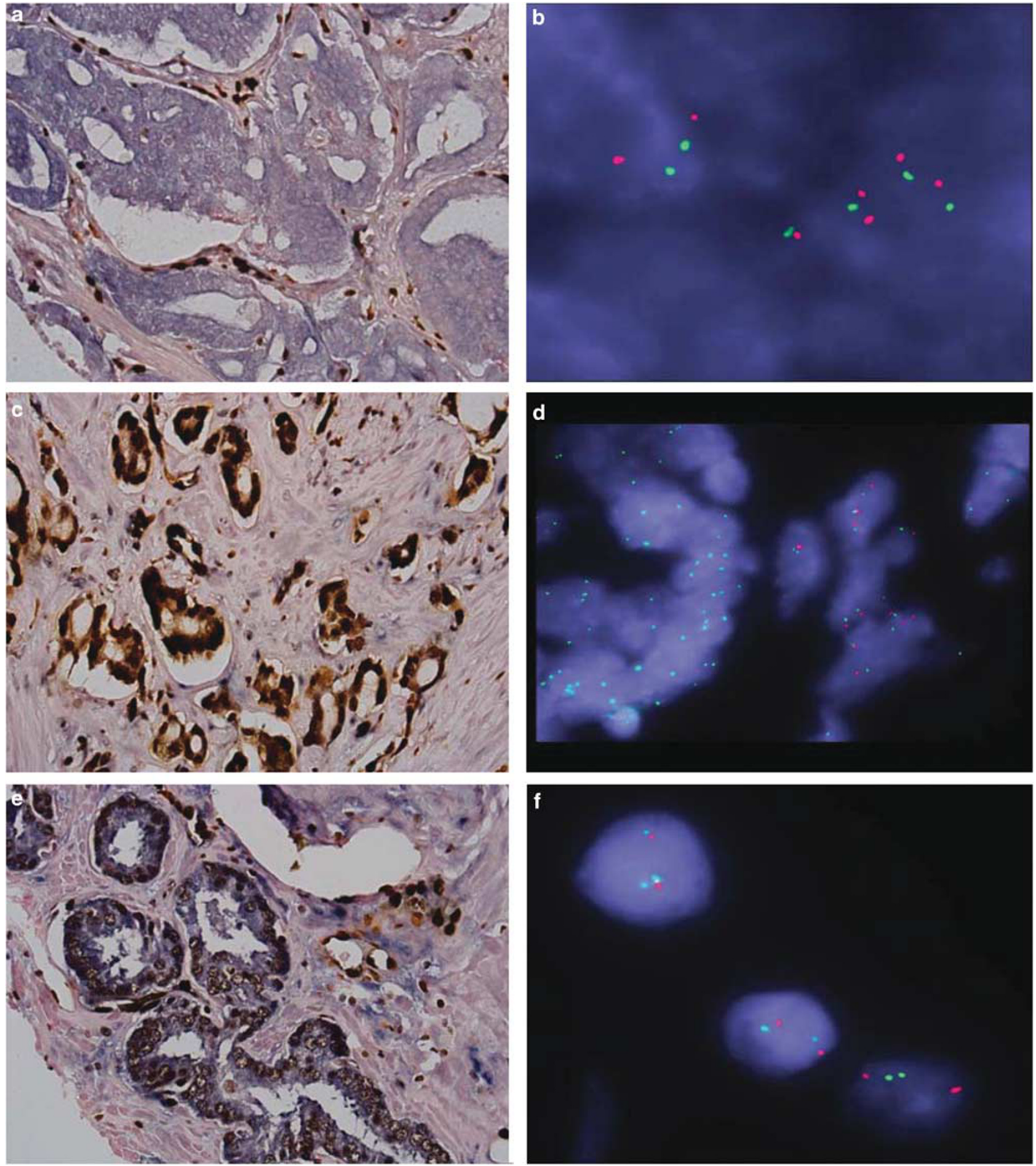

Figure 1 Immunohistochemistry and FISH evaluation of ERG and PTEN in prostate cancer tissues. (a) ERG-PTEN dual immunohistochemisty showing negative ERG and strong PTEN expression (blue) in a prostate cancer sample. Note the positive internal control for ERG antibody staining in the surrounding lymphocytes and endothelial cells $(\times 40)$. (b) Corresponding FISH image showing two copies of normal PTEN signal (red) and two copies of chromosome 10 control probe (green). (c) Positive expression of ERG and negative expression of PTEN antibodies in a prostate cancer sample $(\times 40)$. (d) FISH analysis showing PTEN deletion (loss of red signal) in cancer tissue and adjacent benign prostatic acini showing normal PTEN signal (red) and two copies of chromosome 10 control probe (green). (e) Prostate cancer expressing ERG antibody in the nucleus and PTEN in the cytoplasm $(\times 40)$ and corresponding FISH image showing normal signal pattern for PTEN and chromosome 10 control probe (f). (g) Negative ERG and PTEN expression in a prostate cancer sample $(\times 40)$. (h) FISH demonstrating homozygous loss of PTEN (loss of red signal). (i) Image showing negative expression of ERG and PTEN $(\times 40)$, but FISH confirming heterozygous PTEN deletion $(\mathbf{j})$. 

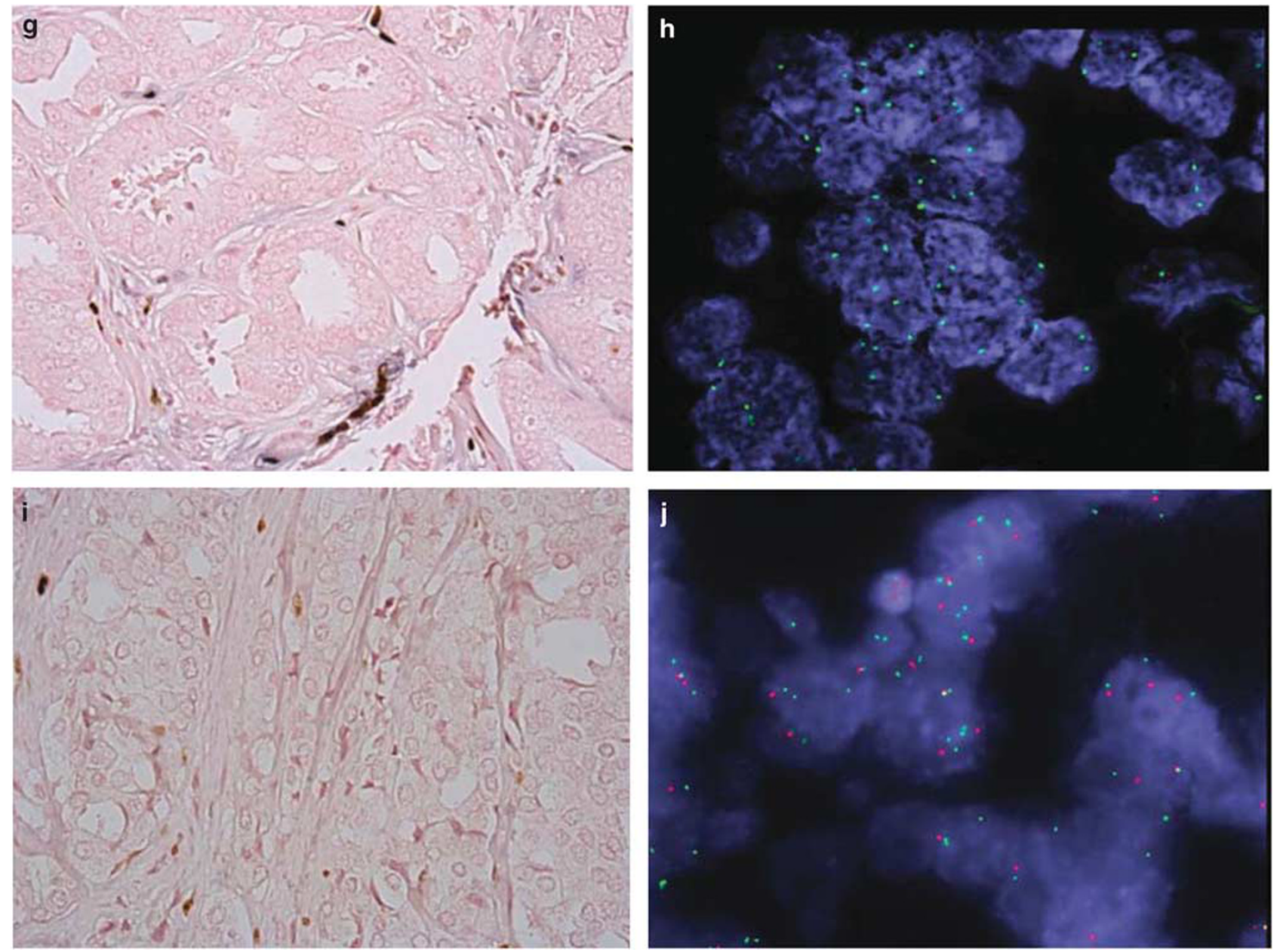

Figure 1 Continued.

Table 4 Types of TMA and distribution of cases for ERG-SPINK1 study

\begin{tabular}{lc}
\hline Types of tissue microarray & $\begin{array}{c}\text { Number } \\
\text { of patients }\end{array}$ \\
\hline Low PSA PCA & 55 \\
Localized PCA & 26 \\
Rare morphology PCA including metastatic & 51 \\
Progression including metastatic PCA & 13 \\
Metastatic PCA & 89 \\
\hline
\end{tabular}

discussed below. Intratumoral heterogeneity was observed for SPINK1 protein expression, even within a given core on a tissue microarray. Some malignant glands expressed SPINK1 protein, whereas adjacent malignant glands were entirely negative (Figure 2e and f). Positive SPINK1 expression was observed in $18(9 \%)$ patients including 4 localized and 14 metastatic carcinomas.

An interesting finding was noted in a prostatectomy sample with limited tumor represented on a tissue microarray (Figure 3a). Some prostate cancer glands showed expression of ERG; however, one gland present partially toward the edge of the biopsy
Table 5 ERG and SPINK1 status

\begin{tabular}{|c|c|c|c|}
\hline & $\begin{array}{l}\text { Localized } \\
\quad \text { PCA }\end{array}$ & $\begin{array}{l}\text { Metastatic } \\
\quad P C A\end{array}$ & $\begin{array}{l}\text { Total no. } \\
\text { of cases }\end{array}$ \\
\hline Distribution of cases & 228 & 56 & 284 \\
\hline ERG positive & $95(42 \%)$ & $16(29 \%)$ & $111(39 \%)$ \\
\hline SPINK1 positive & $12(5 \%)$ & $14(25 \%)$ & $26(9 \%)$ \\
\hline $\begin{array}{l}\text { ERG positive and SPINK1 } \\
\text { positive in single focus }\end{array}$ & $1(0.5 \%)$ & 0 & $1(0.5 \%)$ \\
\hline $\begin{array}{l}\text { ERG positive and SPINK1 } \\
\text { positive in separate foci }\end{array}$ & $1(0.5 \%)$ & 0 & $1(0.5 \%)$ \\
\hline
\end{tabular}

was SPINK1 positive (Figure 3b). We further explored the patient's resection with ERG-SPINK1 immunohistochemisty. As seen on the tissue microarray, the tumor showed expression of both ERG and SPINK1 in adjacent, neighboring foci of the same tumor (Figure 3c). Additionally, in another case of localized prostate cancer we observed a concomitant expression of ERG and SPINK1 in the same focus of the tumor (Figure 3d and e). Because of the advantages of this novel dual immunohistochemisty procedure, to the best of our knowledge, this is the first observation of a rare subset of prostate cancer with concomitant rearrangement of ERG 

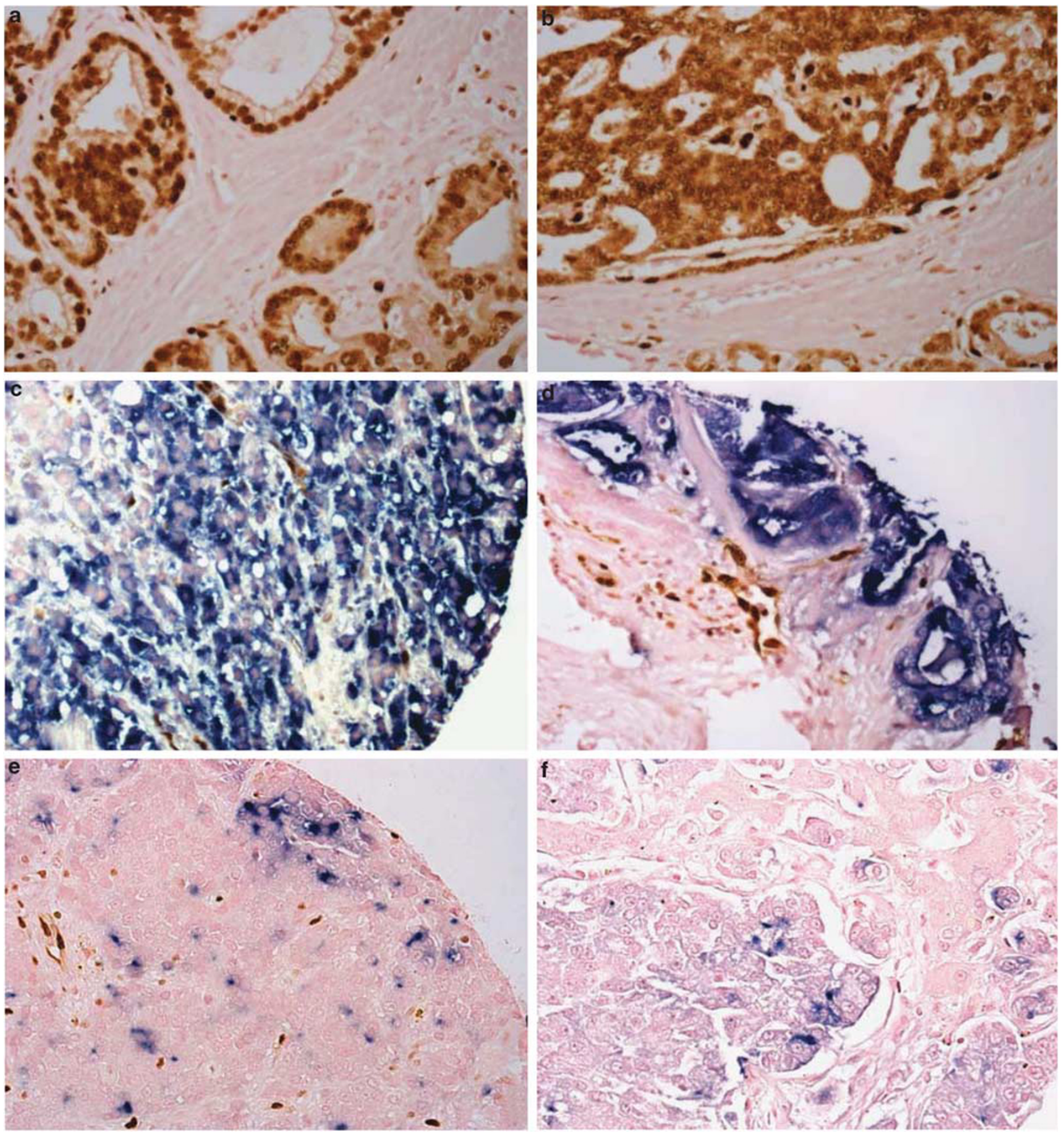

Figure 2 Evaluation of ERG and SPINK1 by dual immunohistochemistry in prostate cancer. Prostate cancer tissues with positive expression of ERG and no expression of SPINK1 $(\times 40)(\mathbf{a}, \mathbf{b})$. ERG and SPINK1 dual immunohistochemisty in a prostate cancer tissue demonstrating diffuse SPINK1 (blue) (c, d) expression and no expression of ERG (brown) (immunohistochemisty, $\times 40$ ). Heterogeneous pattern of SPINK1 expression in minute cancer foci (immunohistochemisty, $\times 40$ ). Note ERG staining of endothelial cells, used as positive internal control $(\mathbf{e}, \mathbf{f})$.

and expression of SPINK1 either in the same or different cancer foci.

\section{Discussion}

Identification of prognostic molecular biomarkers is critical for the clinical management of prostate cancer. With recent improvements in early detection of prostate cancer, studies are now focused on the identification and detection of significant molecular markers that can effectively distinguish men with high-risk disease from the majority of indolent tumors. Recurrent gene fusions involving ETS family genes are observed in a majority of human prostate cancers, the most common being 

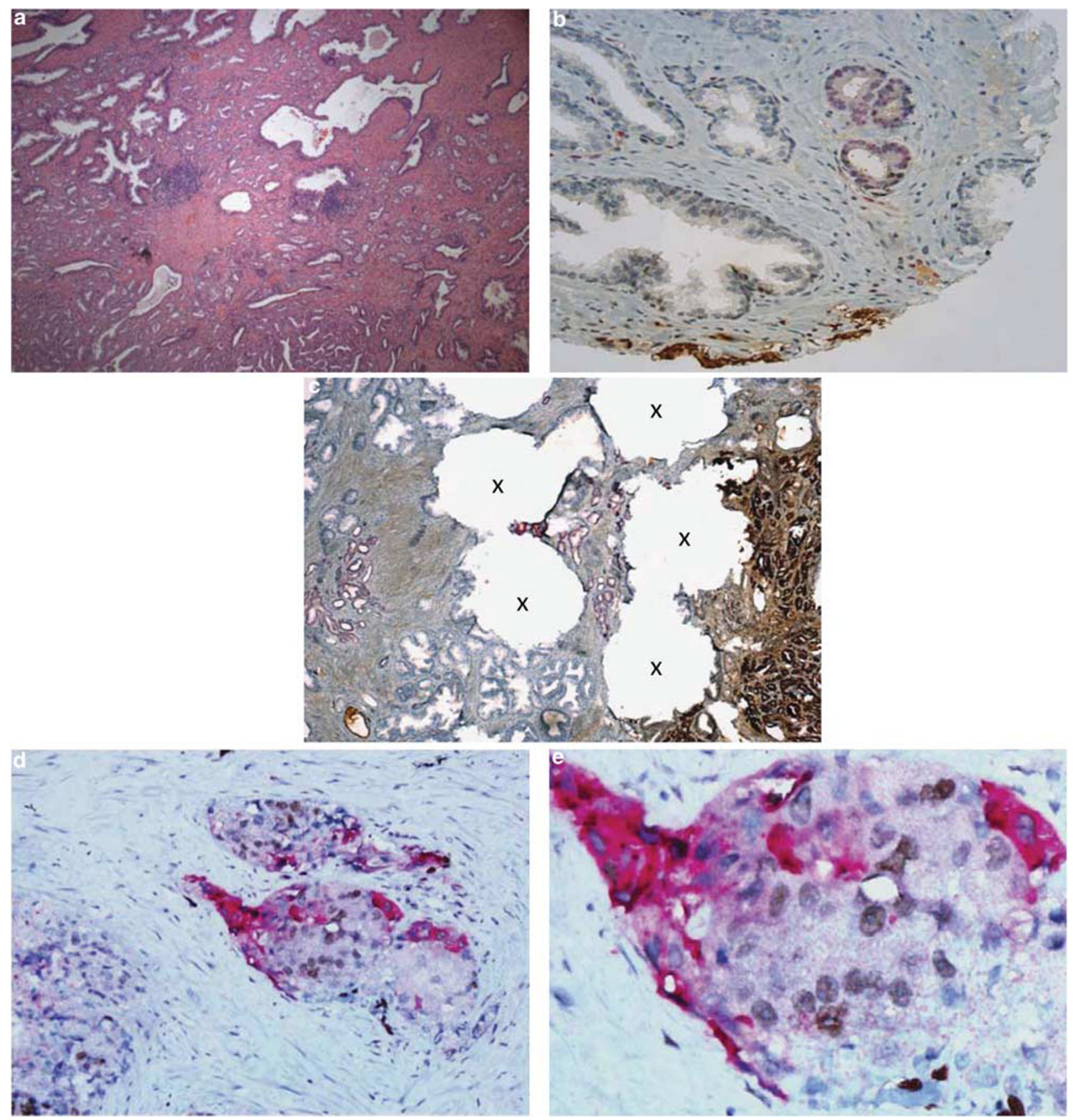

Figure 3 Concomitant expression of ERG and SPINK1 in prostate cancer. Hematoxylin and eosin staining of a prostate cancer tissue (a) with expression of both ERG (red) and SPINK1 (brown) in two different adjacent foci (b). Image from the resection of the same case shown in (b) demonstrating ERG and SPINK1 positivity in adjacent foci with punch holes (X) for tissue microarray biopsies (c). Concomitant expression of ERG (brown) and SPINK1 (red) in the same tumor foci (d, e).

TMPRSS2-ERG fusions occurring in $\sim 50 \%$ of localized prostate cancers, and have potential implications for diagnosis, prognosis and therapy. ${ }^{1,8,9,31}$

In this study we developed dual-color immunohistochemical method for the simultaneous detection of ERG-PTEN and ERG-SPINK1 status in prostate cancer. Previous studies have confirmed the diagnostic utility of immunohistochemisty in identifying ERG rearrangement, ${ }^{46,47,52,53}$ PTEN deletion status ${ }^{48,49,54}$ and SPINK1 overexpression in prostate cancer. ${ }^{17}$ This is the first study to report the successful simultaneous evaluation of ERGPTEN and ERG-SPINK1 status in prostate carcinoma using dual-color immunohistochemistry.

In our ERG-PTEN study, 35\% of prostate carcinomas stained positive with ERG antibody. Previous studies have reported ERG antibody expression in $40-50 \%$ of prostate carcinomas, reflecting the 
incidence of ERG gene rearrangement in the Caucasian population. ${ }^{52,55,56}$ We attribute the slightly lower incidence of ERG antibody expression in our cohort to the overrepresentation of samples with a dominant (index) nodule that did not always include the secondary smaller foci in the tissue microarray evaluated here. ERG rearrangement heterogeneity has been documented in previous studies between discrete tumor nodules within the same prostate gland ${ }^{57,58}$ and three cases in our study demonstrated heterogeneous ERG expression. Endothelial cells and lymphocytes stained positive with ERG antibody consistent with previous studies. ${ }^{46,52,53}$

In this new immunohistochemisty assay for PTEN, background benign glands showed robust PTEN staining, whereas the fibromuscular stroma was predominantly negative, although some cases demonstrated weak staining of the stroma. The presence of an internal positive control allowed us to apply a simple dichotomous scoring system for malignant glands as either decreased/absent or normal cytoplasmic PTEN staining. Lotan et a $4^{49}$ applied similar binary scoring system to assess PTEN status on immunohistochemisty and found this system to be highly reproducible, although their study found that PTEN was expressed in benign prostatic glands as well as the stroma. Sangale et a $1^{59}$ evaluated two different scoring systems. The first system was similar to one used in the present study and they reported high reproducibility and concordance between pathologists. The second method was a three-tier system using $0,1+$ and $2+$ scores with endothelial cells as the reference for $2+$ staining; however, they concluded that endothelial cells were an imperfect control as their intensity varied within the same tissue sample. This system took into account the percent of cells staining and intensity; however, the three-tier scheme was not reproducible among pathologists.

A major benefit of our study is that we further validated our PTEN immunohistochemisty results with FISH. We found $87 \%$ concordance rate between immunohistochemisty and FISH with a sensitivity of $90 \%$ and specificity of $87 \%$. Lotan et $a l^{49}$ validated their immunohistochemisty data with FISH on some patients and high-resolution copy number SNP microarray analysis for a separate group of patients for a total of 119 of 376 cases, with a sensitivity of nearly $80 \%$ for FISH and over $80 \%$ for high-resolution SNP array. Yoshimoto et $a l^{54}$ performed immunohistochemisty and FISH on all of their 35 radical prostatectomy specimens and detected PTEN deletion in 24 of 35 prostate carcinoma patients. All 24 positive cases demonstrated variable weak cytoplasmic and/or nuclear PTEN immunoreactivity, thus demonstrating $100 \%$ concordance.

Although immunohistochemisty reliably detected homozygous PTEN deletion, in our study we were unable to consistently separate heterozygous from homozygous deletions. Immunohistochemisty was very sensitive in selecting homozygous deletions in cases with absent staining; however, there was an overlap of homozygous and heterozygous deletions in cases displaying reduced staining.

TMPRSS2-ERG gene rearrangement has been described as a prostate cancer-specific alteration, present in $40-50 \%$ of prostate cancers. ${ }^{8,9,60,61}$ $E R G$ rearrangement status has traditionally been detected by FISH or reverse transcriptase-PCR techniques that are expensive and require fresh frozen tissues for RNA, specialized equipment and expertise. Recent studies have confirmed high correlation between positive ERG immunohistochemisty staining and FISH for ERG gene rearrangements. ${ }^{46,55}$ The sensitivity and specificity for prediction of ERG gene rearrangements using anti-ERG antibodies have been calculated at 95.7 and $96.5 \% .{ }^{46}$

PTEN genomic loss was identified as a driving molecular aberration in prostate cancer almost 15 years ago, ${ }^{62-64}$ and there is a large body of literature investigating the role of PTEN in tumorigenesis, cancer progression and response to cancer therapies. The reported frequency of PTEN deletion is variable between studies. A recent study from our group reported PTEN deletion in $17 \%$ of localized prostate cancers and $54 \%$ of metastatic cancers. ${ }^{48}$ Similarly, a study by Yoshimoto et $a l^{54}$ reported PTEN genomic deletions in $68 \%$ of prostate cancers. Furthermore, they attributed the variation in reported frequency of $P T E N$ deletion in prostate cancer to differences in tissue preparation, stage of disease and the methodology used to detect molecular aberrations. Cases with intact genomic PTEN that results in negative PTEN immunohistochemisty may be because of the posttranslational inactivation and inversion of PTEN region.

PTEN loss is more common in prostate cancer metastases than in primary tumors as reported by three independent studies, with the incidence of PTEN loss of $\sim 50 \% .{ }^{48,65,66}$ Loss of PTEN protein expression in prostate cancer has been correlated with poor prognosis and biochemical recurrence. ${ }^{67,68}$ PTEN inactivation has been demonstrated to play an important role in progression to androgen-independence in prostate cancer. ${ }^{30,69}$ Yoshimoto et $a l^{67}$ suggested that the acquisition of the deletion and concomitant loss of PTEN functional activity at an earlier phase in prostatic oncogenesis is an important determinant of the molecular pathways that govern a more aggressive tumor phenotype. With biochemical recurrence as the end point, their group identified three patient groups using the following genomic markers: (1) 'poor genomic grade' characterized by both PTEN deletion and TMPRSS2-ERG fusion; (2) 'intermediate genomic grade' with either PTEN deletion or TMPRSS2-ERG fusion and (3) 'favorable genomic grade' in which neither rearrangement was present. ${ }^{44}$ 
As reported by multiple studies, PTEN deletion itself represents an aggressive phenotype and in combination with TMPRSS2-ERG fusion is reported to have a 'poor genomic grade'. The development of a dual-color immunohistochemical assay for rapid, simultaneous evaluation of ERG and PTEN status in prostate cancer would enable better prediction of the course of the disease and identification and treatment of patients at higher risk. Simultaneous ERG and PTEN status detection in biopsies allows early detection in men harboring TMPRSS2-ERG rearrangement and PTEN inactivation with limited cancer on biopsy so they can pursue aggressive therapeutic options. This assay would be particularly advantageous for low-yield biopsy material as both ERG and PTEN status could be detected on a single section.

Our current study parallels a recent publication from our group that identified new methods to risk stratify and detect prostate cancer. ${ }^{70}$ The study demonstrated that quantitative measurement of TMPRSS2-ERG fusion transcript in urine in combination with urine prostate cancer antigen 3 (PCA3) improved the performance of the multivariate Prostate Cancer Prevention Trial (PCPT) risk calculator in predicting cancer on biopsy. Given that ERG rearrangement is present in a subset of high-grade prostatic intraepithelial neoplasia, the dual immunohistochemical ERGPTEN assay may be helpful in risk stratifying the high-grade prostatic intraepithelial neoplasia on biopsies in those cases where high-grade intraepithelial neoplasia was the only significant finding. Future studies exploring this group will be required to assess the utility of dual ERG-PTEN status for risk stratification in high-grade intraepithelial neoplasia cases.

SPINK1 outlier expression was observed in $\sim 10 \%$ of prostate cancers, is mutually exclusive with ETS gene fusions and was found to be associated with an aggressive outcome. ${ }^{17} \mathrm{~A}$ subsequent study suggested that SPINK1 in ETS-negative prostate cancers may be a promising therapeutic target. ${ }^{18}$ Hence, simultaneous detection of ERG rearrangement and SPINK1 status is important not only for molecular categorization of prostate carcinoma and identification of patients with a more aggressive outcome, but also for identifying cancer patients who may benefit from emerging therapeutics. As discussed above, until recently ERG gene rearrangement status was assessed using FISH or reverse transcription-PCR techniques. Recent studies utilizing novel anti-ERG monoclonal antibodies established the strong correlation between ERG gene rearrangement and positive immunohistochemisty staining. ${ }^{46,55}$ Considering the combined incidence of $\sim 50-60 \%$ of prostate cancer with ERG rearrangement and SPINK1 overexpression, we developed a rapid, reliable and simple immunohistochemical assay to simultaneously detect ERG and SPINK1 status of prostate cancer. This assay has clinical implications for early detection in biopsy and prostatectomy specimens. We optimized the new protocol on both needle biopsy (data not shown) and prostatectomy samples.

SPINK1 positivity was detected in $9 \%$ of the cases (26 of 284 patients) consistent with our previous study. ${ }^{17}$ No SPINK1 expression was noted in benign glandular tissue. We confirmed our earlier observation of mutual exclusivity of SPINK1 expression and ETS fusion status. ${ }^{17,18}$ However, we report for the first time two cases with concomitant expression of ERG and SPINK1; in one case in the same focus of tumor and in the other in adjacent foci of same tumor nodule. These cases may represent a rare molecular subtype of prostate cancer and future studies in a large cohort are needed to explore the actual incidence and clinical significance of this subtype. Such observations can be attributed to the advantages of the new dual immunohistochemisty procedure presented in this study.

Although we performed the dual ERG-PTEN and ERG-SPINK1 immunohistochemisty assays using automated protocols, these assays are not restricted to automation; they can also be performed manually. The advantages of automated dual immunohistochemisty assays include simplicity, rapid turnaround and consistency. The dual immunohistochemisty procedure takes on an average $4-5 \mathrm{~h}$ and would be of great value to high-volume laboratories in achieving fast turnaround time. Ours is the first study to report automated dual ERG-PTEN and ERG-SPINK1 immunohistochemisty to simultaneously detect ERG-PTEN and ERG-SPINK1 status in prostate carcinoma. These assays are simple, reliable, reproducible and easily portable to other laboratories. Antibody-based detection of PTEN and ERG shows a high concordance with FISH that offers a reliable alternative method for evaluating their status in prostate cancer. Similarly, we demonstrate that dual ERG-SPINK1 immunohistochemical assay is reproducible and highly sensitive for detecting small foci of SPINK1 expression. These assays will be useful for early screening for prostate cancer to select high-risk patients for targeted therapies based on ERG, PTEN and SPINK1 status. The dual-staining methodology eliminates the need to perform the stain on two separate sections that can be of great value when biopsy samples are limited. Validation studies of this dual immunohistochemisty in prostate needle biopsies are underway. The assays have utility in retrospective or prospective studies for risk stratification of prostate cancer as well as for prognostic and therapeutic decision-making purposes. Future studies using this novel dual immunohistochemistry assay will help to identify the incidence of the newly identified rare molecular subsets of prostate cancer with ERG-SPINK1 expression in the same or independent foci. 


\section{Acknowledgements}

This work was supported in part by the US National Institutes of Health Early Detection Research Network (U01 CA111275 and U01 CA113913), NIH S.P.O.R.E. (P50 CA69568) and R01 CA132874. NP is supported by a University of Michigan Prostate SPORE Career Development Award, and AMC is supported by the Howard Hughes Medical Institute, the Doris Duke Foundation and the Prostate Cancer Foundation and is an American Cancer Research Professor and a Taubman Scholar. We thank Karen Giles and Jyoti Athanikar for critical reading and submission process of this manuscript.

\section{Disclosure/conflict of interest}

AMC serves on the advisory boards of Gen-Probe, and Ventana Medical Systems/Roche. AMC and SAT are co-inventors on a patent filed by the University of Michigan covering the diagnostic and therapeutic field of use for ETS fusions in prostate cancer. The diagnostic field of use has been licensed to GenProbe. Gen-Probe and Ventana/Roche did not play a role in the design and conduct of this study, in the collection, analysis, or interpretation of the data or in the preparation, review or approval of the article. NP does receive research funding from Ventana/ Roche but this funding did not play a part in development of the assay. SAT has consulted for and received honoraria from Ventana/Roche. JMM holds a sponsored research agreement with Ventana/ Roche, but this funding did not play a part in development of the assay. KC, CC and GP are employees of Ventana/Roche and provided reagents and technical help for the development of this assay. The remaining authors declare no conflict of interest.

\section{References}

1 Tomlins SA, Rhodes DR, Perner S, et al. Recurrent fusion of TMPRSS2 and ETS transcription factor genes in prostate cancer. Science 2005;310:644-648.

2 Tomlins SA, Mehra R, Rhodes DR, et al. TMPRSS2:ETV4 gene fusions define a third molecular subtype of prostate cancer. Cancer Res 2006;66: 3396-3400.

3 Helgeson BE, Tomlins SA, Shah N, et al. Characterization of TMPRSS2:ETV5 and SLC45A3:ETV5 gene fusions in prostate cancer. Cancer Res 2008;68:73-80.

4 Hermans KG, Bressers AA, van der Korput HA, et al. Two unique novel prostate-specific and androgenregulated fusion partners of ETV4 in prostate cancer. Cancer Res 2008;68:3094-3098.

5 Attard G, Clark J, Ambroisine L, et al. Heterogeneity and clinical significance of ETV1 translocations in human prostate cancer. Br J Cancer 2008;99:314-320.

6 Tomlins SA, Laxman B, Dhanasekaran SM, et al. Distinct classes of chromosomal rearrangements create oncogenic ETS gene fusions in prostate cancer. Nature 2007;448:595-599.

7 Han B, Mehra R, Dhanasekaran SM, et al. A fluorescence in situ hybridization screen for E26 transformation-specific aberrations: identification of DDX5-ETV4 fusion protein in prostate cancer. Cancer Res 2008;68:7629-7637.

8 Kumar-Sinha C, Tomlins SA, Chinnaiyan AM. Recurrent gene fusions in prostate cancer. Nat Rev Cancer 2008;8:497-511.

9 Clark JP, Cooper CS. ETS gene fusions in prostate cancer. Nat Rev Urol 2009;6:429-439.

10 Mosquera JM, Perner S, Genega EM, et al. Characterization of TMPRSS2-ERG fusion high-grade prostatic intraepithelial neoplasia and potential clinical implications. Clin Cancer Res 2008;14:3380-3385.

11 Cerveira N, Ribeiro FR, Peixoto A, et al. TMPRSS2ERG gene fusion causing ERG overexpression precedes chromosome copy number changes in prostate carcinomas and paired HGPIN lesions. Neoplasia 2006;8:826-832.

12 Clark J, Merson S, Jhavar S, et al. Diversity of TMPRSS2-ERG fusion transcripts in the human prostate. Oncogene 2007;26:2667-2673.

13 Perner S, Demichelis F, Beroukhim R, et al. TMPRSS2:ERG fusion-associated deletions provide insight into the heterogeneity of prostate cancer. Cancer Res 2006;66:8337-8341.

14 Perner S, Mosquera JM, Demichelis F, et al. TMPRSS2ERG fusion prostate cancer: an early molecular event associated with invasion. Am J Surg Pathol 2007;31:882-888.

15 Mehra R, Tomlins SA, Yu J, et al. Characterization of TMPRSS2-ETS gene aberrations in androgen-independent metastatic prostate cancer. Cancer Res 2008;68:3584-3590.

16 Tomlins SA, Bjartell A, Chinnaiyan AM, et al. ETS gene fusions in prostate cancer: from discovery to daily clinical practice. Eur Urol 2009;56:275-286.

17 Tomlins SA, Rhodes DR, Yu J, et al. The role of SPINK1 in ETS rearrangement-negative prostate cancers. Cancer Cell 2008;13:519-528.

18 Ateeq B, Tomlins SA, Laxman B, et al. Therapeutic targeting of SPINK1-positive prostate cancer. Sci Transl Med 2011;3:72ra17.

19 Palanisamy N, Ateeq B, Kalyana-Sundaram S, et al. Rearrangements of the RAF kinase pathway in prostate cancer, gastric cancer and melanoma. Nat Med 2010;16:793-798.

20 Di Cristofano A, Pandolfi PP. The multiple roles of PTEN in tumor suppression. Cell 2000;100:387-390.

21 Besson A, Robbins SM, Yong VW. PTEN/MMAC1/ TEP1 in signal transduction and tumorigenesis. Eur J Biochem 1999;263:605-611.

22 Goberdhan DC, Wilson C. PTEN: tumour suppressor, multifunctional growth regulator and more. Hum Mol Genet 2003;12 Spec No 2:R239-R248.

23 Datta SR, Dudek H, Tao X, et al. Akt phosphorylation of BAD couples survival signals to the cell-intrinsic death machinery. Cell 1997;91:231-241.

24 Cardone MH, Roy N, Stennicke HR, et al. Regulation of cell death protease caspase-9 by phosphorylation. Science 1998;282:1318-1321.

25 Ashcroft M, Ludwig RL, Woods DB, et al. Phosphorylation of HDM2 by Akt. Oncogene 2002;21:1955-1962.

26 Majumder PK, Febbo PG, Bikoff R, et al. mTOR inhibition reverses Akt-dependent prostate intrae- 
pithelial neoplasia through regulation of apoptotic and HIF-1-dependent pathways. Nat Med 2004; 10:594-601.

27 Brunet A, Bonni A, Zigmond MJ, et al. Akt promotes cell survival by phosphorylating and inhibiting a Forkhead transcription factor. Cell 1999;96:857-868.

28 Graff JR, Konicek BW, McNulty AM, et al. Increased AKT activity contributes to prostate cancer progression by dramatically accelerating prostate tumor growth and diminishing p27Kip1 expression. J Biol Chem 2000;275:24500-24505.

29 Koksal IT, Dirice E, Yasar D, et al. The assessment of PTEN tumor suppressor gene in combination with Gleason scoring and serum PSA to evaluate progression of prostate carcinoma. Urol Oncol 2004;22: 307-312.

30 Bertram J, Peacock JW, Fazli L, et al. Loss of PTEN is associated with progression to androgen independence. Prostate 2006;66:895-902.

31 Demichelis F, Fall K, Perner S, et al. TMPRSS2:ERG gene fusion associated with lethal prostate cancer in a watchful waiting cohort. Oncogene 2007;26: 4596-4599.

32 Rajput AB, Miller MA, De Luca A, et al. Frequency of the TMPRSS2:ERG gene fusion is increased in moderate to poorly differentiated prostate cancers. J Clin Pathol 2007;60:1238-1243.

33 Mehra R, Tomlins SA, Shen R, et al. Comprehensive assessment of TMPRSS2 and ETS family gene aberrations in clinically localized prostate cancer. Mod Pathol 2007;20:538-544.

34 Cheville JC, Karnes RJ, Therneau TM, et al. Gene panel model predictive of outcome in men at high-risk of systemic progression and death from prostate cancer after radical retropubic prostatectomy. J Clin Oncol 2008;26:3930-3936.

35 Nam RK, Sugar L, Wang Z, et al. Expression of TMPRSS2:ERG gene fusion in prostate cancer cells is an important prognostic factor for cancer progression. Cancer Biol Ther 2007;6:40-45.

36 Nam RK, Sugar L, Yang W, et al. Expression of the TMPRSS2:ERG fusion gene predicts cancer recurrence after surgery for localised prostate cancer. Br J Cancer 2007;97:1690-1695.

37 Dai MJ, Chen LL, Zheng YB, et al. [Frequency and transcript variant analysis of gene fusions between TMPRSS2 and ETS transcription factor genes in prostate cancer]. Zhonghua Yi Xue Za Zhi 2008;88:669-673.

38 Rouzier C, Haudebourg J, Carpentier X, et al. Detection of the TMPRSS2-ETS fusion gene in prostate carcinomas: retrospective analysis of 55 formalin-fixed and paraffin-embedded samples with clinical data. Cancer Genet Cytogenet 2008;183:21-27.

39 Gopalan A, Leversha MA, Satagopan JM, et al. TMPRSS2-ERG gene fusion is not associated with outcome in patients treated by prostatectomy. Cancer Res 2009;69:1400-1406.

40 Yoshimoto M, Joshua AM, Chilton-Macneill S, et al. Three-color FISH analysis of TMPRSS2/ERG fusions in prostate cancer indicates that genomic microdeletion of chromosome 21 is associated with rearrangement. Neoplasia 2006;8:465-469.

41 Lapointe J, Kim YH, Miller MA, et al. A variant TMPRSS2 isoform and ERG fusion product in prostate cancer with implications for molecular diagnosis. Mod Pathol 2007;20:467-473.
42 Darnel AD, Lafargue CJ, Vollmer RT, et al. TMPRSS2ERG fusion is frequently observed in Gleason pattern 3 prostate cancer in a Canadian cohort. Cancer Biol Ther 2009;8:125-130.

43 Saramaki OR, Harjula AE, Martikainen PM, et al. TMPRSS2:ERG fusion identifies a subgroup of prostate cancers with a favorable prognosis. Clin Cancer Res 2008;14:3395-3400.

44 Yoshimoto M, Joshua AM, Cunha IW, et al. Absence of TMPRSS2:ERG fusions and PTEN losses in prostate cancer is associated with a favorable outcome. Mod Pathol 2008;21:1451-1460.

45 Rubin MA, Maher CA, Chinnaiyan AM. Common gene rearrangements in prostate cancer. J Clin Oncol 2011;29:3659-3668.

46 Park K, Tomlins SA, Mudaliar KM, et al. Antibodybased detection of ERG rearrangement-positive prostate cancer. Neoplasia 2010;12:590-598.

47 Falzarano SM, Zhou M, Carver P, et al. ERG gene rearrangement status in prostate cancer detected by immunohistochemistry. Virchows Arch 2011;459:441-447.

48 Han B, Mehra R, Lonigro RJ, et al. Fluorescence in situ hybridization study shows association of PTEN deletion with ERG rearrangement during prostate cancer progression. Mod Pathol 2009;22:1083-1093.

49 Lotan TL, Gurel B, Sutcliffe S, et al. PTEN protein loss by immunostaining: analytic validation and prognostic indicator for a high risk surgical cohort of prostate cancer patients. Clin Cancer Res 2011;17:6563-6573.

50 Leinonen KA, Tolonen TT, Bracken H, et al. Association of SPINK1 expression and TMPRSS2:ERG fusion with prognosis in endocrine-treated prostate cancer. Clin Cancer Res 2010;16:2845-2851.

51 Han B, Mehra R, Suleman K, et al. Characterization of ETS gene aberrations in select histologic variants of prostate carcinoma. Mod Pathol 2009;22:1176-1185.

52 Yaskiv O, Zhang X, Simmerman K, et al. The utility of ERG/P63 double immunohistochemical staining in the diagnosis of limited cancer in prostate needle biopsies. Am J Surg Pathol 2011;35:1062-1068.

$53 \mathrm{He} \mathrm{H,} \mathrm{Magi-Galluzzi} \mathrm{C,} \mathrm{Li} \mathrm{J,} \mathrm{et} \mathrm{al.} \mathrm{The} \mathrm{diagnostic} \mathrm{utility}$ of novel immunohistochemical marker ERG in the workup of prostate biopsies with "atypical glands suspicious for cancer”. Am J Surg Pathol 2011;35: 608-614.

54 Yoshimoto M, Cutz JC, Nuin PA, et al. Interphase FISH analysis of PTEN in histologic sections shows genomic deletions in $68 \%$ of primary prostate cancer and $23 \%$ of high-grade prostatic intra-epithelial neoplasias. Cancer Genet Cytogenet 2006;169:128-137.

55 Furusato B, Tan SH, Young D, et al. ERG oncoprotein expression in prostate cancer: clonal progression of ERG-positive tumor cells and potential for ERG-based stratification. Prostate Cancer Prostatic Dis 2010;13:228-237.

56 Chaux A, Albadine R, Toubaji A, et al. Immunohistochemistry for ERG expression as a surrogate for TMPRSS2-ERG fusion detection in prostatic adenocarcinomas. Am J Surg Pathol 2011;35:1014-1020.

57 Mehra R, Han B, Tomlins SA, et al. Heterogeneity of TMPRSS2 gene rearrangements in multifocal prostate adenocarcinoma: molecular evidence for an independent group of diseases. Cancer Res 2007;67:7991-7995.

58 Barry M, Perner S, Demichelis F, et al. TMPRSS2-ERG fusion heterogeneity in multifocal prostate cancer: clinical and biologic implications. Urology 2007;70:630-633. 
59 Sangale Z, Prass C, Carlson A, et al. A robust immunohistochemical assay for detecting PTEN expression in human tumors. Appl Immunohistochem Mol Morphol 2011;19:173-183.

60 Esgueva R, Perner S, LaFargue JC, et al. Prevalence of TMPRSS2-ERG and SLC45A3-ERG gene fusions in a large prostatectomy cohort. Mod Pathol 2010;23: 539-546.

61 Scheble VJ, Braun M, Beroukhim R, et al. ERG rearrangement is specific to prostate cancer and does not occur in any other common tumor. Mod Pathol 2010;23:1061-1067.

62 Cairns P, Okami K, Halachmi S, et al. Frequent inactivation of PTEN/MMAC1 in primary prostate cancer. Cancer Res 1997;57:4997-5000.

$63 \mathrm{Li} \mathrm{J}$, Yen C, Liaw D, et al. PTEN, a putative protein tyrosine phosphatase gene mutated in human brain, breast, and prostate cancer. Science 1997;275: 1943-1947.

64 Steck PA, Pershouse MA, Jasser SA, et al. Identification of a candidate tumour suppressor gene, MMAC1, at chromosome 10q23.3 that is mutated in multiple advanced cancers. Nat Genet 1997;15:356-362.
65 Suzuki H, Freije D, Nusskern DR, et al. Interfocal heterogeneity of PTEN/MMAC1 gene alterations in multiple metastatic prostate cancer tissues. Cancer Res 1998;58:204-209.

66 Taylor BS, Schultz N, Hieronymus H, et al. Integrative genomic profiling of human prostate cancer. Cancer Cell 2010;18:11-22.

67 Yoshimoto M, Cunha IW, Coudry RA, et al. FISH analysis of 107 prostate cancers shows that PTEN genomic deletion is associated with poor clinical outcome. Br J Cancer 2007;97:678-685.

68 Bedolla R, Prihoda TJ, Kreisberg JI, et al. Determining risk of biochemical recurrence in prostate cancer by immunohistochemical detection of PTEN expression and Akt activation. Clin Cancer Res 2007;13: 3860-3867.

69 Jiao J, Wang S, Qiao R, et al. Murine cell lines derived from Pten null prostate cancer show the critical role of PTEN in hormone refractory prostate cancer development. Cancer Res 2007;67:6083-6091.

70 Cao Q, Mani RS, Ateeq B, et al. Coordinated regulation of polycomb group complexes through microRNAs in cancer. Cancer Cell 2011;20:187-199.

Supplementary Information accompanies the paper on Modern Pathology website (http://www.nature.com/ modpathol) 